\title{
Synchronization of Estrus in Cattle: A Review
}

\author{
R. Islam \\ Division of Animal Reproduction, Gynaecology \& Obstetrics \\ Faculty of Veterinary Sciences \& Animal Husbandry, \\ S. K. University of Agricultural Sciences \& Technology (K), Srinagar - 190006, J \& K., India. \\ E-mail: rafiqvet@gmail.com
}

\begin{abstract}
Numbers of estrus synchronization programmes are available in cattle based on the use of various hormones like progesterone, prostaglandin F2a and their various combinations with other hormones like estrogen and Gonadotrophin Releasing hormone $(\mathrm{GnRH})$. Selection of appropriate estrus synchronization protocol should be made on the basis of management capabilities and expectations of the farmer. Synchronization of oestrus can be accomplished with the injection of prostaglandin F2a alone, but it needs proper detection of the ovarian status of the cows as prostaglandin F2a is active in only functional corpus luteum in between 8 to 17 days of estrous cycle. Progesterone may reduce fertility up to 14 percent, but short time progesterone exposure (less than 14 days) is beneficial. Addition of GnRH in the Progesterone or Prostaglandin based synchronization programme is helpful for more synchrony in estrus as GnRH may be helpful to synchronize the oestrous cycle in delayed pubertal heifers and post partum cows (Post partum anoestrum) and further a single, timed artificial insemination is possible with this method. New methods of synchronizing estrus in which the GnRH-PG protocol is preceded by progesterone treatment offer effective synchronization of estrus with high fertility.
\end{abstract}

Keywords: Estrus, Synchronization, Hormone, Prostaglandin, Progesterone, Anoestrus, Estrogen, Gonadotrophin Hormone.

\section{I ntroduction}

Synchronization of estrus implies the manipulation of the estrous cycle or induction of estrus to bring a large percentage of a group of females into estrus at a short, predetermined time (Odde, 1990). Synchronization of estrus is one of the advanced managemental process through which the humane errors and managemental costs could be minimized. It is particularly beneficial in Sheep, where timely heat detection is difficult due to exhibitions of less external heat symptoms and also in large cattle herd. Several authors described the experiments that have been conducted since the discovery of ovarian steroids and which have lead to the effective control of the length of the bovine estrous cycle and the timing of estrus and ovulation (Hansel and Schechter, 1972; Hansel and Beal 1979; Patterson et al., 1989; Odde, 1990; Larson and Ball, 1992). Synchronization of estrus helps in fixing the breeding time within a short predefined period and thereby scheduling the parturition time at the most favourable season, when the newborns can be reared in suitable environment with ample food for enhancing their survivability. Fertility in farm animals may be expected towards higher side as timely breeding of the animals is possible with this technique. Estrus synchronization provides more economic returns by improving the production efficiency in animals.

Instead of females being bred over a 21-day period, synchronization can shorten the breeding period to less than five days, depending on the treatment regimen. Scientific management of cows and calves is possible within the limited resources due to the friendly environment. Production of uniform calf crop for the future replacement in the farm is another important advantage of this programme. In migratory herd of cattle maintained by nomads, synchronization of oestrus may improve the production potential by inseminating the animals with superior germ plasm within a predefined short period at low altitudes before or after the animals reared in summer grazing at alpine pastures available on the great Himalaya.

The current and future direction of estrous synchronization is to focus on combining traditional methods of controlling cycle length with the manipulation of follicular development. The immediate goal of controlling both CL function and follicular development is to devise a treatment that 
will synchronize estrus more precisely and to control the time of ovulation more exactly to allow a single, timed insemination without the need for detection of behavioral estrus. Combination of $(\mathrm{GnRH})$ with the Prostaglandin F2a (Prusley et al., 1994; Prusley et al., 1995 a, b) and Progesterone (Patterson et al., 1999) based synchronization programme has shown a new direction in the estrus synchronization of cattle with the manipulation of follicular development. Knowledge on the hormonal profile and functional structures present in the ovaries at various stages of estrous cycle is very much important for the selection and successful implementation of the estrus synchronization programme.

\section{Basic approach for estrus synchronization}

Basic approach is to control the timing of the onset of estrus by controlling the length of the estrous cycle. The various approaches for controlling cycle length are:

1. Administration of Prostaglandin to regress the corpus luteum $(\mathrm{CL})$ of the animal before the time of natural luteolysis, or

2. Administration of Progesterone or more commonly synthetic progestins to temporarily suppress the ovarian activity, or

3. A newer way of creating estrous synchrony is by using gonadotropin-releasing hormone $(\mathrm{GnRH})$ or an analogue, which causes ovulation of a large follicle. This help in synchronizing estrous cycle in anoestrous female.

\section{Methods of Estrus Synchronization}

\section{Prostaglandin treatment}

One of the oldest ways to synchronize estrus is by using a luteolytic agent such as Prostaglandin F2a, or an analogue, which causes the regression of the corpus luteum (Lauderdale, 1972; Louis et al., 1972; Rowson et al., 1972; King and Robertson, 1974; Roche, 1977). Prostaglandin F2a is only effective if administered between days 8 to 17 of the estrous cycle when functional corpus luteum is available in one of the ovaries. King et al. (1982) and others (Tanabe and Hahn, 1984; Stevenson et al., 1984; Watts and Fuquay, 1985) demonstrated that cattle injected with PGF2a between Days 5 and 9 of the cycle were less responsive than those injected later in the cycle. Fertility is high following prostaglandin synchronization. Most studies indicate that conception rates are similar for beef cows or heifers synchronized with PGF2a and those bred after a naturally-occurring heat. A conception rate of $59 \%$ for synchronized cows and
$62 \%$ for untreated cows has been reported when cows were bred $12 \mathrm{~h}$ after estrus (Moody and Lauderdale, 1977). Synchrony of estrus and fertility with these products is good with cyclic females, such as virgin heifers, but can not induce estrous cycles in noncycling cows.

(a) One Shot Prostaglandin : A single injection of prostaglandin is given to cyclic females, and then these females are bred as they express estrus. The disadvantage of this program is that one-third of the females do not respond to the injection. The programme may be modified first with the detection of estrus in the cows of the herd for 5 days and inseminated the cows showing estrus and only rest of the cows are given a single injection of Prostaglandin. This represents the greatest savings in cost and labor associated with treatments because only one injection is given and not all the cows need it.

(b) Two Shot Prostaglandin : Two injections of prostaglandins are given at an interval of 10 to 14 days (Wiltbank et al., 1967; Inskeep, 1973; Cooper, 1974) once stage of estrous cycle in the cows is unknown. Crossbred and non descript cows in India were treated with two injections of Lutalyse (Dinoprost thromithamine) $25 \mathrm{mg}$ intramuscularly at 11 days apart and reported 80.00 percent estrus rate in non descript and $100 \%$ in crossbred cows (Sahatpure and Patil, 2008).

Two injections of prostaglandin are given 10 to 12 days apart. Detection of estrus is not required before or between injections. All cycling cows should respond to the second injection regardless of what stage of the estrous cycle they were in when the first injection was administered. The programme may be modified with the breeding of all females exhibiting estrus subsequent to the first PGF2a injection. Then the second injection is given only to the females that were not bred. This option lowers expense and handling, but results in two synchronized groups instead of one and a longer breeding period.

\section{Progesterone treatment}

Synchronization of estrus with progestogens (Nellore and Cole, 1956) maintains high levels of progesterone in the female's system, even after the regression of the corpus luteum. Synchrony of estrus occurs 2 to 5 days following progestin removal. Commercial products that fall into this category are melengesterol acetate (oral feeding), Syncro-Mate-B (Ear Implant) and CIDR (Intra-vaginal device). Estrus was synchronized in only $48 \%$ of the cows treated on $d$ 3 , but the synchronization was $100 \%$ when treatment began on d 9 of the estrous cycle (Pratt et al., 1991). In 
general, the longer the progestin was administered to cattle, the higher rate of estrous synchronization, but the lower the fertility of the synchronized animals. Simultaneously, Kaltenbach et al. (1964) and others (Loy et al., 1960; Wiltbank, 1966) demonstrated that estradiol was luteolytic when administered early in the bovine estrous cycle. Hence, combining progestin treatment with the administration of estradiol at the initiation of treatment enabled the period of progestin treatment to be shortened (9-14 d) without reducing the percentage of animals exhibiting a synchronized estrus. This treatment regimen was the basis for the commercial product SYNCRO-MATE B marketed in the USA, as well as the PRID (Progesterone-releasing intravaginal device) and EAZI-BREED CIDR (Controlled intra-vaginal drug release device) marketed in Europe, Australia and New Zealand. The treatment of cyclic cows or heifers with exogenous progestin preceded by an injection of estradiol is usually followed by a high incidence ( $>90 \%)$ of estrus during the 5 days following progestin removal.

Several workers successfully used ultrasonography to demonstrate that progestin administration at "sub-luteal" levels inhibits estrus and ovulation and synchronizes estrus in cattle, but that a persistent, estrogen-secreting follicle develops when progestin treatment extends the estrous cycle (Lucy et al., 1990; Sirous and Fortune, 1990; Cupp et al., 1992). Development of the persistent follicle is caused by increased pulsatile secretion of gonadotropins during the period when the exogenous progestin is inhibiting estrus, but the corpus luteum has regressed (Kojima et al., 1992; Savio et al., 1993; Custer et al., 1994). The low fertility of cows bred at the synchronized estrus following long-term administration of progestin is due to premature resumption of meiosis of ova or abnormal development of embryos derived from ova of persistent follicles (Wishart and Young, 1974; Mihm et al., 1994; Ahmad et al., 1995; Revah and Butler, 1996). The pregnancy rate of cows bred following progestin treatment that caused a persistent, dominant follicle to ovulate was reduced by 17 to $35 \%$ (Sanchez et al., 1993; Savio et al., 1993; Wehrmann et al., 1993). Treatment of cattle with progestogens for less than $14 \mathrm{~d}$ was reported not to reduce conception percentage (Wiltbank et al., 1968; Roche, 1974; Roche, 1976). In addition, short-term exposure to progestogens causes some anestrus (postpartum or pre pubertal) cattle to begin cycling. However, for these short-term progestogen systems to be effective in synchronizing estrus, a luteolytic agent must be incorporated.

\section{Techniques of Progesterone treatment} (a) Melengesterol Acetate (MGA) feeding : Option I: MGA is added to feed such that females receive $0.5 \mathrm{mg}$ per head per day for 14 days. Upon removal of MGA from the feed, cyclic females begin to show estrus. This estrus is subfertile, and it is not recommended to breed. Females should be bred on the second estrus following MGA removal. The administration of MGA at the recommended daily rate of $0.5 \mathrm{mg}$ prevents the expression of behavioural estrus, blocks the preovulatory surge of $\mathrm{LH}$, and ovulation (Imwalle et al., 2002).

Option II : An injection of prostaglandin is given 1519 days after removal of MGA from the feed (Brown et al., 1988; Larson et al., 1996; Jaeger et al., 1992). Brown et al. (1988) developed a system in which MGA was fed for $14 \mathrm{~d}$ and PGF2a was administered 16 to 18 $\mathrm{d}$ after the last day of MGA feeding. This system was designed to place cattle in the late luteal phase of the estrous cycle at the time of PGF2a administration. This system initially synchronizes estrus within the $7 \mathrm{~d}$ following the last MGA feeding (Patterson et al., 1989; Odde, 1990, King and Odde 1993). The administration of PGF2a $17 \mathrm{~d}$ after the last MGA feeding causes the timing of PGF2a to occur after Day 10 of the estrous cycle in the majority of cattle and thus the estrous response and fertility of cattle are expected to be maximized as PGF2a is more effective during the late luteal phase ( $\mathrm{d} 10$ to 15 ) than during the early luteal phase (King et al., 1982; Tanabe and Hann, 1984; Watts and Fuquay, 1985). The rate of synchronization of estrus following MGA-PGF2a is usually greater than that following the use of PGF2a alone (Patterson et al., 1995).

Option III: Two injections of prostaglandin are given; one at the time of MGA removal from the feed and another 15 days following removal. This further reduces time spent in estrous detection and breeding and provides more concentrated synchrony.

\section{(b) Syncro-Mate-B (Ear I mplant)}

SMB treatment late in the estrous cycle (> d 14) in cow gives lower conception rates. The optimum time for SMB treatment to begin is between $\mathrm{d} 8$ and $\mathrm{d}$ 12 of the estrous cycle to maximize estrus response.

\section{(c) Application of CI DR}

This controlled, internal drug- releasing (CIDR) insert for cattle is made by molding a thin layer of silicon and progesterone mixture $(10 \% \mathrm{w} / \mathrm{w})$ around a nylon spine under high temperature. The CIDR contains $1.38 \mathrm{~g}$ progesterone and is designed to maintain elevated blood concentrations of progesterone to at least $2 \mathrm{ng} / \mathrm{ml}$ for up to 10 days. Being relatively thin, the CIDR is easily inserted into 
the vagina and has good retention capacity $(2.5 \%$ loss rate is normal). A flexible nylon tail is attached to the device to allow for easy removal. The CIDR Cattle Insert provides an exogenous source of the hormone progesterone during the 7-day administration period. Removal of the CIDR Cattle Insert on treatment day 7 results in a rapid fall in plasma progesterone levels, which results in synchronization of estrus in those animals responding to treatment.

\section{DR and ProstaglandinF2a}

The CIDR is inserted into the vagina of the female for 7 days. An injection of PGF is to be given 1 day before the CIDR is removed. Females are inseminated based upon detection of estrus during the 3 to 6 day period after CIDR withdrawal. This system should effectively synchronize estrus in a large proportion of cyclic females since treatment for 7 days with the CIDR prevents animals from being in the early stage of the cycle and not responsive to PGF2a induced regression of the CL. Some anestrous females will be induced to ovulate and show heat during the synchronization period. The advantage of CIDR plus PGF treatment than the single PGF injection was that CIDR plus PGF could increase the synchronization rate by approximately $30 \%$, and pregnancy rate by approximately $20 \%$, in both the anestrous and cyclic females (Lucy et al., 2001).

\section{GnRH - Based Synchronization Systems}

Synchrony of estrus and fertility with a combination of GnRH and Prostaglandin F2a are good for cyclic females and this combination may induce cyclicity in cows experiencing postpartum anoestrus (Prusley et al., 1994; Prusley et al., 1995 a,b). This most recently developed synchronization treatments combine traditional methods of controlling cycle length with the manipulation of follicular development in order to "program" or "select" the ovulatory follicle. The new methods synchronize estrus more precisely and control the time of ovulation more exactly in order to allow a single, timed insemination without the need for detection of behavioral estrus.

Administration of GnRH during the bovine estrous cycle causes regression or ovulation of the dominant follicle and initiates the emergence of a new wave of follicular growth an average of $2.5 \mathrm{~d}$ following treatment (Pursley et al., 1995 a). Atresia or ovulation of the dominant follicle depends on the status (growing, static or regressing) of the dominant follicle at the time of GnRH injection (Silcox et al., 1993; Twagiramungu et al., 1994). Ovulation of a growing dominant follicle occurred $100 \%$ of the time following $\mathrm{GnRH}$ administration, however, ovulation of dominant follicles in the static or regressing phases occurred $33 \%$ and $0 \%$ of the time, respectively. Pursley et al. (1995a) reported that the range in timing of ovulation in lactating dairy cows following $\mathrm{GnRH}-$ PGF2a treatment extended from 84 to $120 \mathrm{~h}$ postPGF2a. They demonstrated that the range in ovulation time in cows could be reduced to $8 \mathrm{~h}(72$ to $80 \mathrm{~h}$ postPGF2a) if a second GnRH injection was administered $48 \mathrm{~h}$ after PGF2a in the GnRH-PGF2a treatment (Ovsynch protocol). The four systems for synchronization of estrus with GnRH-PG combinations are OVSYNCH, COSYNCH, SELECT SYNCH and HYBRID SYNCH.

The initial GnRH injection (day 1; GnRH) is used to program follicle growth in cyclic females and to induce ovulation (to provide progestin preexposure) in anestrous females. The PGF2a (PGF; day 8 ) induces regression of CL that are present to cause a decline in progesterone. The second GnRH given on day 10-11 induces ovulation of dominant follicles that have been pre-programmed by the first GnRH treatment. The major GnRH programs that do not involve use of the CIDR are described first in the following section.

(a) GnRH- PGF System: This combination represents the simplest GnRH - based system. A common name for the GnRH - PGF system is "Select Synch".

Select-Synch

Single dose of GnRH and Prostagalndin were injected on day 1 and day 8 , respectively. Some cows (8\%) exhibit estrus up to 48 hours before PGF (d 6). The "early" heats are fertile and cows can be inseminated 12 hours after detection. The peak estrous response occurs 2-3 days after PGF with a range of days $1-5$. With this system, a minimum of 5 days of estrus detection after PGF and 2 days preceding PGF is required to detect most heats.

(b) GnRH - PGF + GnRH System: This system is a GnRH - PGF system that includes a second GnRH injection (+GnRH) given to all, or some cows between 48 and 72 hours after PGF (day 2 to 3 ), with timed AI on all or a portion of the herd. Several variations of this system are being used.

Ovsynch

The Ovsynch program is comprised of an injection of $\mathrm{GnRH}$ on day 1, an injection of prostaglandin on day 8 , a second injection of GnRH on day 10 and then timed insemination on day 11 (Pursley et al., 1994; Pursley et al.,1995; Pursley et al.,1997a,b). Pursley et al. (1995b) indicated that pregnancy rates varied when cows were timed 
inseminated at $0,8,16,24$ or $32 \mathrm{~h}$ after the second injection of GnRH in the Ovsynch program and the highest pregnancy rate $(45 \%)$ was achieved when insemination was done $16 \mathrm{~h}$ after the second GnRH injection. The first GnRH injection alters follicular growth by inducing ovulation of the largest follicle (dominant follicle) in the ovaries after the GnRH injection to form a new or additional CL (Pursley et al., 1995). Thus, estrus usually does not occur until a PGF2a injection regresses the natural $\mathrm{CL}$ and the secondary CL (formed from the follicle induced to ovulate by the first GnRH injection).

Therefore, a new group of follicles appears in the ovaries (based on transrectal ultrasonographic evidence) within 1 to 2 days after the first injection of GnRH (Vasconcelos et al., 1999). From that new group of follicles, a newly developed dominant follicle emerges, matures, and can ovulate after estrus is induced by PGF2a or it can be induced to ovulate after a second injection of GnRH. The GnRH injections release pituitary luteinizing hormone ( $\mathrm{LH})$, the natural ovulation-inducing hormone of the estrous cycle. The stage of the estrous cycle when ovsynch was initiated also affect synchronization and conception rate (Vascon-celos et al., 1999). Higher conception rate was reported in subestrus buffaloes initiating the treatment with Ovsynch during the later stages of estrous cycle, but conception rate was 0 in anestrus buffaloes though incidence of cyclicity was observed due to the treatment (Ravikumar and Asokan, 2008). Moreira et al., (2000) reported that initiating the ovsynch protocol in dairy heifers on day 15 of the estrous cycle exhibited estrus before the second GnRH injection, whereas, no estrus behaviour was observed when the protocol was initiated on $\mathrm{d} 2,5$, 10 (or) 18 of the estrous cycle. Advantages of this program are tight synchronization of estrus, most females respond to the program and it encourages estrus in non-cycling cows that are at least 30 days postpartum.

Co-Synch: The CO-Synch program is comprised of an injection of GnRH on day 1, an injection of prostaglandin on day 8 and then a second injection of GnRH with breeding on day 10 . The advantages are tight synchronization of estrus, most females respond to the program and it encourages estrus in non-cycling cows that are at least 30 days postpartum.

Hybrid-Synch: The Hybrid-Synch program is implemented with an injection of GnRH on day 1, an injection of prostaglandin on day 8 and then estrous detection and breeding from day 8 to 11 . Females not observed in estrus from day 8 to 11 are bred on day 11 and given a second injection of GnRH. This program has a lower cost and less handling compared with Ovsynch and CO-Synch but more than Select-Synch. The primary advantage is that Hybrid-Synch appears to have the highest conception rates among all $\mathrm{GnRH}-$ prostaglandin programs.

(c) Addition of progesterone in the GnRH-PG : MGA-GnRH-PG protocol: MGA is administered to the cows orally for 14 days. Ten days after the withdrawal of MGA an injection of GnRH is given. Seven days after the GnRH injection an injection of PGF2a is given. $80 \%$ of the cows showed estrus within 48 to 96 hours after PGF2a injection (Patterson et al., 1999).

\section{CIDR to GnRH - Based protocol}

Failure to appropriately synchronize cyclic animals or to induce a potentially fertile ovulation in anestrous females can have major influences on the success of a synchronization program. The addition of a CIDR to GnRH - based programs has the potential to reduce losses in each of these areas. The most common use of the CIDR with GnRH - based systems involves insertion of the CIDR on day 1, and withdrawal of the CIDR on day 8. An injection of GnRH is given on day of CIDR insertion. The CIDR is kept in situ for seven days. On the day of CIDR withdrawal, an injection of prostaglandin is given. The second GnRH injection is given after two days of prostaglandin injection.

The primary benefit of inclusion of the CIDR in GnRH-based programs is that it guarantees that females will be exposed to progesterone during the period between day 1 and day 8 . This progesterone exposure will result in normal (21 days) rather than short (10 days) cycles in previously anestrous cows. Furthermore, since the withdrawal of a progestin has been demonstrated to induce onset of cycles in some anestrous females, the likelihood of an ovulation (either spontaneous or in response to second GnRH injection) is enhanced. A second benefit to inclusion of the CIDR in GnRH-based programs is that the early heats (days 6 to day 9) that are inherent to these systems are prevented. The progesterone released by the CIDR, will prevent estrus and ovulation between days 1 and 9. The females that show early heats in GnRH - based programs would have conception rates of near $0 \%$ if timed AI is performed on day 10 or 11 .

\section{Managemental intervention}

Management in general has tremendous role in reproduction of animals. Appropriate nutritional management of the herd is essential for successful implementation of several synchronization programs in both cows and heifers. Managemental procedures 
like timed insemination and calf removal have been reported to be beneficial for synchronization of estrus and may also be applied in most of the synchronization programs for better results.

Timed I nsemination: Generally conception rates on timed insemination are lower than for visual observation. In a large trial using 45 herds, Fogwell et al. (1986) recorded a $22 \%$ lower conception rate when a single, timed breeding at $80 \mathrm{hr}$ post-PGF2a was compared to breeding $12 \mathrm{hr}$ after detecting a synchronized estrus. However, this lower conception rate may be offset by the reduction in management from timed insemination.

48-hour Calf Removal: Frequency of suckling by calves causes a hormonal response that inhibits return to estrus (suckling inhibition). It is evident in beef cows. Many studies have concluded that short-term calf removal combined with other forms of synchronization improves estrus synchrony and conception rates in cows. Even 48-hour calf removal alone has been shown to cause synchrony and cyclicity in some cows. This procedure is useful, but requires increased management and good facilities to prevent separated cows and calves from rejoining each other.

\section{References}

1. Ahmad, N.; F. N. Schrick; R. L. Butcher and E. K. Inskeep (1995): Biol. Reprod., 52:1129-1135.

2. Brown, L. H., et. al. (1988): Theriogenology 30: 1-12.

3. Cooper, M. J. (1974): Vet. Rec., 95: 200-203.

4. Cupp, A.,et.al. (1992): Biol. Reprod., 45: (Suppl 1):106.

5. Custer, E. E.,et.al.(1994): J. Anim. Sci., 72:1282- 1289.

6. Fogwell, R. L., et. al. (1986): J. Dairy Sci., 69:1665-1672.

7. Hansel, W. and R. J. Schechter (1972): Biotechnical procedures concerning the control of theestrous cycle in domestic animals. VIIth Int. Cong. Anim. Reprod. Artif. Insem. Munich., 1:78-96.

8. Hansel, W. and W. E. Beal (1979): Ovulation control in cattle. In: BARC Symposia III. Anim. Reprod. p. 91. Allanheld, Osmun \& Co., Montclair, NJ.

9. Imwalle, D. B.; D.1. Fernandez and K.K. Schillo (2002): J. Anim. Sci., 80: 1280-1284.

10. Inskeep E. K. (1973): J. Anim. Sci., 36: 1149-1157.

11. Jaeger, J. R. ,et.al. (1992): J. Anim. Sci., 70: 2622- 2627.

12. Kaltenbach, C. C.,et. al. (1964): J. Anim. Sci., 23: 995-1001.

13. King, G. J. and H. A. Robertson (1974): Theriogenology, 1:123-128.

14. King, M. E. , et. al. (1982): Theriogenology, 18:191-200.

15. King, M. E.; and K. G. Odde (1993): MGA-prostaglandin synchronization system: where we have come from and where we are going. In: Proc. Beef Improv. Fed. 25th Res. Symp. and Ann. Meet., p 1. Beef Improvement Federation, Colby, KS.

16. Kojima, N., et. al. (1992): Biol. Reprod., 47:1009-1017.

17. Larson, L. L.; and P. J. H. Ball (1992): Theriogenology, 38:255-267.
18. Larson, R. L.; L. R. Corah; C. W. Peters (1996): Theriogenology, 45: 851-863.

19. Lauderdale, J. W. (1972): J. Anim. Sci., 35:246 (Abstr.).

20. Louis, T. M.; H. D. Hafs and D. A. Morrow (1972): J. Anim. Sci., 35:247 (Abstr.).

21. Loy, R. G.; R. G. Zimbelman, and L. E. Casida (1960): $J$. Anim. Sci., 19:175-182.

22. Lucy, M. C. W. W. Thatcher and K. L. Macmillan (1990): Theriogenology, 34:325-340.

23. Lucy, M.C., et. al. (2001): J. Anim. Sci., 79:982-995.

24. Mihm, M., et. al. (1994): J. Reprod. Fertil., 13:14 (Abstr.).

25. Moody, E. L. and J. W. Lauderdale (1977): J. Anim. Sci. 45(Suppl. 1):189 (Abstr.).

26. Pratt, S. L. J. C. Spitzer; G. L. Burns and B. B. Plyler (1991): J. Anim. Sci., 69:2721-2726.

27. Moreira, F.; R. L. De la sota; T. Diaz and W. W. Thatcher (2000): J.Anim. Sci., 78: 1568.

28. Nellore, J.E. and H.H.Cole (1956): J. Anim. Sci 15: 650-661.

29. Odde, K. J. (1990): J. Anim. Sci., 68:817-830.

30. Patterson, D. J., et.al. (1989): J. Anim. Sci., 67:1895-1906.

31. Patterson, D. J., et. al.(1995): J. Anim. Sci., 73:954-959.

32. Patterson, D. J.; F. N. Kojima and M. F. Smith (1999): $J$. Anim. Sci., 77 (Suppl. I). 220 (Abstr.)

33. Prusley J. R.; M. O. Mee; M. D. Brown and M. C. Wilt Bank (1994): J. Dairy Sci., 77 (Suppl 1): 230 (Abstr).

34. Pursley, J. R.; M. O. Mee and M. C. Wiltbank (1995a): Theriogenology, 44:915-923.

35. Pursley, J. R.; R. W. Silcox and M. C. Wiltbank (1995b): $J$ Dairy Sci., 78:(Suppl. 1)279 (Abstr.).

36. Pursley, J. R.; M. R. Kosorok and M. C. Wiltbank(1997a): $J$. Dairy Sci., 80:301-306.

37. Pursley, J. R., et. al. (1997b): J. Dairy Sci. 80:295-300.

38. Ravikumar, K. and S. A. Asokan (2008): Indian Vet. J., 85: 388-392.

39. Revah, I. and W. R. Butler(1996): J. Reprod. Fertil., 106:39-47.

40. Roche, J. F. (1974): J Reprod Fertil., 37:135-138.

41. Roche, J. F. (1976): J. Reprod. Fertil., 40:433-440.

42. Roche, J. F. (1977): Vet. Res. Com., 1:121-129

43. Rowson, L. E.; A, R. Trevit and A. Brand (1972): J. Reprod. Fertil., 29:145-154.

44. Sahatpure, S.K. and M.S. Patil (2008): Vet. World, 1(7): 203-204.

45. Sanchez, T., et. al.(1993): Biol. Reprod., 49:1102-1107.

46. Savio, J. D., et. al. (1993): J. Reprod. Fertil., 97:197-203.

47. Silcox, R. W. K. L. Powell and T. E. Kiser (1993): J. Anim. Sci., 71: (Suppl. 1) 513 (Abstr.).

48. Sirois. J. and J.E. Fortune (1990): Endocrinology, 127:916-925.

49. Stevenson, J. S.; M. K. Schmidt and E. P. Call (1984): J. Dairy Sci., 67:1798-1805.

50. Tanabe, T. Y. and R. C. Hahn (1984): J. Anim. Sci., 58:805-811

51. Twagiramungu, H.; L. A. Guilbault and J. G. Proulx (1994): J.Anim. Sci., 72:1796-1805.

52. Vasconcelos, J.L.M., et. al.(1999): Theriogenology, 52:1067-1078.

53. Watts, T. L. and J. W. Fuquay (1985): Theriogenology, 23:655-661.

54. Wehrman, M. E., et. al. (1993): Biol. Reprod., 49:214-220.

55. Wiltbank, J. N. (1966): J. Reprod. Fertil., (Suppl. 1):1-8.

56. Wiltbank, J. N. and C. W. Kasson (1968): J. Anim. Sci., 27:113-116.

57. Wishart, D. F. and I. M. Young (1974): Vet. Rec., 95:503-508. 